\title{
Maser Emission from Icy Planets Orbiting Young Stars
}

\author{
Viacheslav Slysh \\ Arecibo Observatory, HC3 Box 53995, Arecibo PR 00612, USA \\ Astro Space Center, Profsoyuznaya St. 84/32, 117997 Moscow, Russia
}

\begin{abstract}
A model of maser emission spots as icy planets orbiting young massive stars is proposed. It is argued that the interstellar gas phase abundance of methanol, $\mathrm{H}_{2} \mathrm{O}$ and $\mathrm{OH}$ is much too low to produce maser emission from small gas clumps. The interstellar grains, on the other hand, consisting mainly of water ice and several per cent of methanol, can produce a favorable environment for the maser emission when evaporated. Big comets or planets covered by the water/methanol ice orbiting young massive stars can be the sites of the maser emission. The ice evaporates under stellar radiation, and water and methanol molecules emit corresponding maser lines, while $\mathrm{OH}$ molecules are produced from dissociation of water by the stellar radiation. VLBI observations of masers in $\mathrm{W} 3(\mathrm{OH}), \mathrm{W} 75 \mathrm{~N}$ and V645 Cyg show that proposed planets are found in disks, and are moving on Keplerian orbits around $\mathrm{O}, \mathrm{B}$-stars. In the case of the O-star in $\mathrm{W} 3(\mathrm{OH})$ the planets are located beyond the compact HII region of the star. Extended envelopes of the icy planets provide molecular abundance, gas temperature and density adequate for the maser emission.
\end{abstract}

\section{Introduction}

Methanol, $\mathrm{OH}$ and water masers are generally seen toward star-forming regions. Here we do not consider masers associated with evolved stars and circum-nuclear regions in active galaxies. The presence of a proto-star or massive young star is manifested by a source of infrared emission re-radiated by a dense dust envelope or by the ultra-compact HII region if the young star is sufficiently hot. The standard model of the masers considers them as originating in dense gas clumps possibly compressed and heated by a bow shock or by the expanding HII region which surrounds a young massive star. The methanol and water abundance in the clumps may be enhanced by the evaporation of dust grain mantles. In order to provide sufficiently high maser gain the clumps are assumed to be in the form of cylinders aligned along the line of site. Another model assumes that there are no such clumps, and the masers are observed from turbulent molecular clouds along favorable lines of sight where a coherent amplification is possible. Both these models fail to predict the observed brightness of the masers mainly because the necessary gain can not be achieved with a relatively low abundance of water and methanol in the interstellar medium. In this communication we 


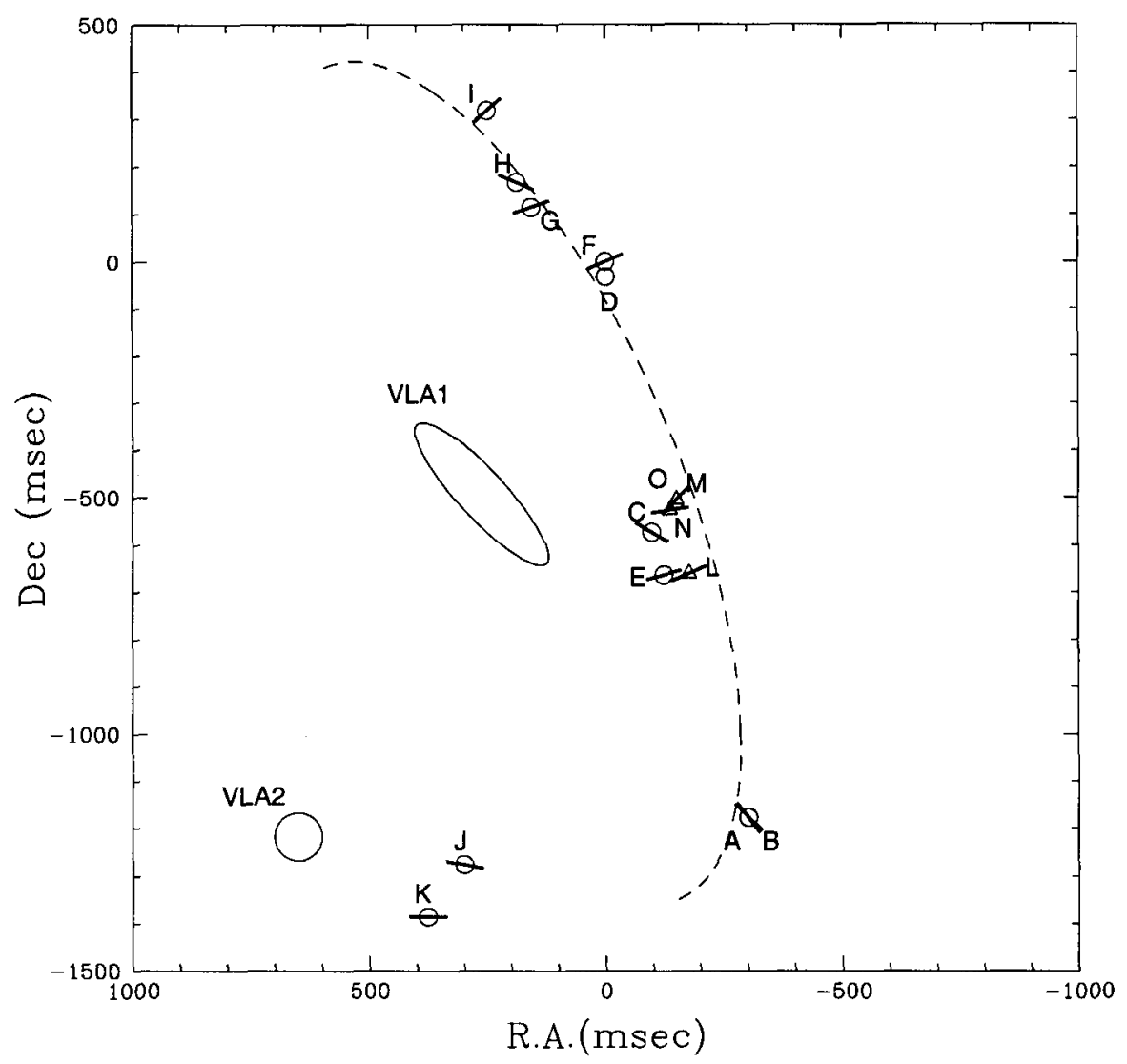

Figure 1. $\mathrm{OH}$ maser spots and ultra-compact $\mathrm{HII}$ regions in $\mathrm{W} 75 \mathrm{~N}$

show that recent VLBI data on masers require still higher molecular abundances, and propose a new site for the maser emission: gaseous envelopes of small icy planets orbiting proto-stars and young stars.

\section{High Angular Resolution Maser Maps}

$\mathrm{OH}$ and methanol maser spots in $\mathrm{W} 75 \mathrm{~N}$ are located on a portion of an arc surrounding apparently the exciting young massive star which is visible through the ultra-compact HII region VLA1 (Fig. 1). Another group of the maser spots is related to the ultra-compact HII region VLA2 (Slysh et al. 2002b). The star of VLA1 has a luminosity $1.4 \times 10^{5} \mathrm{~L}_{\odot}$ which corresponds to a $20 \mathrm{M}_{\odot}$ O9 ZAMS star. VLA2 is excited by a slightly lower mass star. The radius of the arc shown by a dashed line is about $2000 \mathrm{AU}$. The angular size of the maser spots is less than 1 mas, or $2 \mathrm{AU}$. 


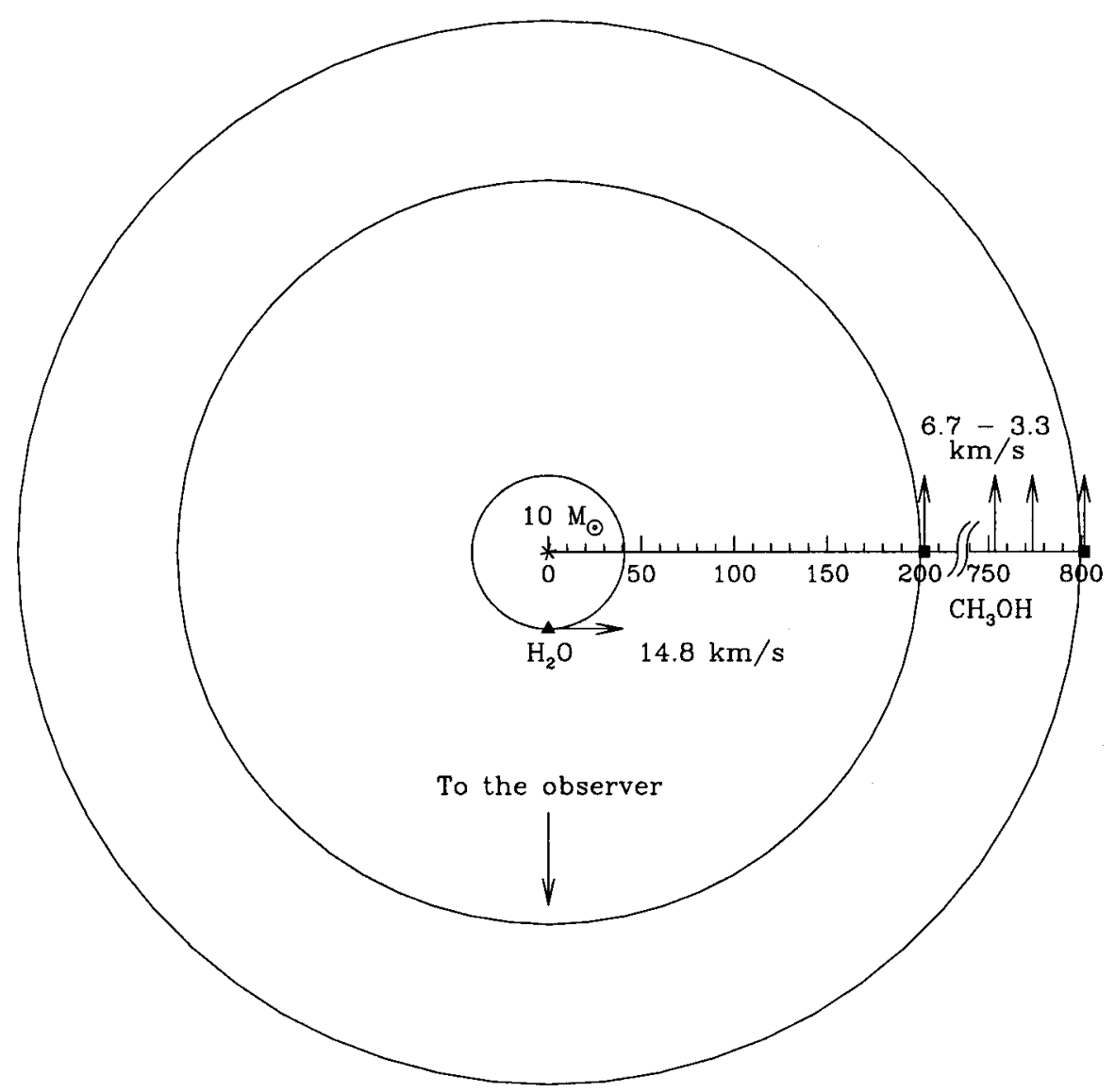

Figure 2. Model of methanol and $\mathrm{H}_{2} \mathrm{O}$ masers in a disc around V645 Cyg. Distance from the star is in AU.

W $3(\mathrm{OH})$ is another ultra-compact HII region, which is excited by a $50 \mathrm{M}_{\odot}$ star and surrounded by a cloud of $\mathrm{OH}$ and methanol maser spots. The spots are located roughly on a belt of radius $2000 \mathrm{AU}$ (Menten et al. 1992; Slysh et al. 1999).

V645 Cyg = RAFGL 2789 is a source of maser emission in $\mathrm{OH}$, methanol and water lines. It has no visible ultra-compact HII region, but from the infrared luminosity of $2.5 \times 10^{5} \mathrm{~L}_{\odot}$ one can derive a mass for the young star or proto-star of 10 to $20 \mathrm{M}_{\odot}$. From the VLBI maps of methanol and $\mathrm{H}_{2} \mathrm{O}$ masers a model has been produced which is shown in Fig. 2 (Slysh et al. 2002a). (Details of the VLBI observations of this source can be found in the paper by Val'tts et al. on page 279 in this volume.) There are numerous maps of other methanol masers showing linear or arc structure in the distribution of maser spots (Norris et al. 1998; Minier, Booth, \& Conway 2000). 


\section{Methanol Maser Models}

Methanol masers require as a pump far-infrared radiation which can be supplied from the hot dust re-emitting radiation of the central star. Temperature of the dust is about $150 \mathrm{~K}$. A cold gas with a temperature lower than the temperature of dust is also needed as a stock by collisions. For the sufficient gain the column density of methanol molecules divided by the line width must be about $10^{12}$ (Sobolev, Cragg, \& Godfrey 1997). For the typical line width of masers of $0.3 \mathrm{~km} \mathrm{~s}^{-1}$, the methanol column density in the masers must be $3 \times 10^{16} \mathrm{~cm}^{-2}$. The size of the methanol maser spots is less or equal to several astronomical units (AU) as follows from VLBI measurements. If the transverse size measured by the VLBI is the same as the line of sight size, that is about $5 \times 10^{13} \mathrm{~cm}$, then the density of methanol molecules must be $600 \mathrm{~cm}^{-3}$. On the other hand, the total particle density has to be less than $3 \times 10^{7} \mathrm{~cm}^{-3}$ in order not to quench maser emission by collisions. This means a methanol abundance higher than $2 \times 10^{-5}$, which can be compared with the interstellar methanol abundance of $10^{-9}$. The required methanol abundance in masers is a factor $2 \times 10^{4}$ higher than in the general interstellar medium, or in molecular clouds, where the methanol is produced by gas phase chemical reactions. In shocks the methanol abundance can be enhanced by a factor up to 100 due to an additional supply from dust grains heated by the shock. But it is still a factor of 200 short of the required methanol abundance in masers. Two solutions of this problem can be proposed. The first solution is that accepted in current maser models: beaming of the maser emission toward the observer. This is not a geocentric or heliocentric model assuming that all the masers direct their emission in narrow beams toward us. The masers are assumed to have a shape of long cylinders, and the maximum maser gain is along the cylinder axis. We see only those cylinders whose axis is oriented towards us. Other cylinders are seen closer to the normal of the cylinder axis, with low maser gain, and they are invisible from the Earth. Their maximum emission is in other directions, away from us. In order to provide the observed maser intensity the cylinders must have length to width ratios of at least 200 if the methanol abundance is a hundred fold increased with respect to the interstellar abundance. Another solution of the methanol abundance problem is to look for an environment other than clumps of interstellar gas. Such an environment could be clouds of evaporated ice mantles of interstellar dust grains. The ice is known to consist of frozen water with high content of methanol alcohol. Comets have a similar chemical composition. Evaporated gas contains water and methanol, and no molecular hydrogen which could collisionally quench the masers, as in the interstellar gas.

\section{Interstellar Grains and Comets}

Infrared spectra of some star-forming regions show strong absorption bands of water ice and methanol. These bands are attributed to the absorption by interstellar dust grains which have silicate cores covered by ice mantles. In the direction of the high-mass proto-stars RAFGL 7009S and W 33A, the abundance of methanol relative to water is 30 per cent and 5 to 22 per cent, respectively (Dartois et al. 1999). In the direction of NGC 7539 IRS 9, the methanol abun- 
dance is 25 per cent (Schutte et al. 1996). In the direction of RAFGL 2136 the abundance of methanol is 8.7 per cent (Skinner et al. 1992). However, in other directions the methanol abundance is less than several per cent (Dartois et al. 1999). In dense molecular clouds where star formation is taking place, dust grains stick together and form large bodies. This process is probably most intense in cold disks around proto-stars or young stars. Comets are believed to be formed in such a way. This scenario is supported by the chemical composition of comets which is very similar to the chemical composition of interstellar dust grains. In particular infrared spectra of comets show water ice, methanol and $\mathrm{CO}$ as major constituents. For comet Halley 1986, the spectral information is confirmed by Giotto's mass-spectrometer in situ measurements of the chemical composition (Eberhardt et al. 1994). Microwave spectra of some comets show rotational lines of methanol and $\mathrm{OH}$ (product of dissociation of water) in the gaseous phase, after evaporation from the nucleus (Bockelee-Morvan et al. 1994). Estimated abundance of methanol relative to water in comets is between 0.8 per cent for comet Levy 1980 and 8 per cent for comet Swift-Tuttle 1992. It is not known if the difference in abundance between different comets is real or results from a different heliocentric distance during the measurements. Comets move on elliptical or hyperbolic orbits, and significant evaporation of ice from their cores occurs only at the closest distance to the Sun. Evaporated gas escapes from the surface due to the low gravity of comet nuclei and an extended envelope or coma is formed. Water molecules are dissociated, and free $\mathrm{OH}$ molecules are formed. Such envelopes have favorable conditions for the maser emission: high abundance of molecules, low content of quenching hydrogen molecules, availability of the pumping of far-infrared emission from the star. The only reason why masers are not found in the Solar System comets is the low column density of molecules because of the small size of the nuclei.

\section{Cometary (Planetary) Maser Model}

The Solar System comets are small now but there might have existed bigger comets in the past, which have totally evaporated during the lifetime of the Solar System. It is natural to assume that other stars also have their comets including young stars and proto-stars. Young stars and protostars may have comets of a much bigger size. Evaporation of ice from the surface of such big comets will create extended gaseous envelopes around them, with the molecular column density sufficient for the maser emission. In order to estimate the size of the envelope sufficient to provide the typical maser gain, the methanol column density should be not less than $3 \times 10^{16} \mathrm{~cm}^{-2}$. The water molecule density should not exceed $3 \times 10^{7} \mathrm{~cm}^{-3}$ in the region of maser amplification, and this gives the amplification length $10^{10} \mathrm{~cm}$, assuming a methanol to water density ratio (abundance) of 0.1 . We assume that the gravity of the comets is low enough to allow water and methanol molecules to escape freely with their thermal velocity of about $1 \mathrm{~km} \mathrm{~s}^{-1}$. At the distance from the surface $10^{10} \mathrm{~cm}$ where the $\mathrm{H}_{2} \mathrm{O}$ density is $10^{7} \mathrm{~cm}^{-3}$ the outflow rate of water molecules is $4 \times 10^{33} \mathrm{~s}^{-1}$, which is four orders of magnitude higher than the outflow rate from Solar System comets. In order to produce such an outflow rate the proposed extrasolar comets must have a correspondingly larger surface area. If a Solar System comet of radius 
$10 \mathrm{~km}$ produces $5 \times 10^{29} \mathrm{~s}^{-1}$ water molecules from its surface, then a proposed extra-solar comet, in order to produce a four orders of magnitude larger outflow, must have a radius of $900 \mathrm{~km}$. This is a small enough body which has low gravity and does not hold evaporated gas in an atmosphere. Instead, the evaporated gas is continuously expanding into outer space. In this respect the body resembles a comet. On the other hand, it is large enough to be called a planet. The closest analog in the Solar System is probably a Kuiper Belt Object. At the distance of 800 to $2000 \mathrm{AU}$ from a central star, and with a luminosity of about $3 \times 10^{5} \mathrm{~L}_{\odot}$, the temperature of the surface will be about $250 \mathrm{~K}$, which is high enough to evaporate ice. The radiation from the star re-emitted by circumstellar dust in the infrared and far-infrared will serve as a pump for the masers. The comets/planets are moving around the star on Keplerian orbits. At their distance, the Keplerian velocity is about 3 to $5 \mathrm{~km} \mathrm{~s}^{-1}$ which is very close to the observed velocity spread of maser emission features. The orbital period is about 20,000 years. The mass loss due to ice evaporation is quite high, about $10^{11} \mathrm{~g} \mathrm{~s}^{-1}$, and the planet loses most of its mass in several million years, or in about 100 orbits (planetary 'years'). This model is based on the observed and model parameters of methanol masers. $\mathrm{OH}$ masers are similar to the methanol masers in that the gas density and far-infrared emission needed are of the same order of magnitude, so that the model must equally well apply to $\mathrm{OH}$ masers. $\mathrm{H}_{2} \mathrm{O}$ masers require a two orders of magnitude higher gas density, and a several times higher temperature. They may arise in planets located closer to the star as shown in the model of V645 Cyg (Fig. 2).

\section{References}

Bockelee-Morvan, D., Crovisier, J., Colom, P., \& Despois, D. 1994, A\&A, 287, 647

Dartois, E., Schutte, W., Geballe, T. R., Demyk, K., Ehrenfreund, P., \& D'Hendecourt, L. 1999, A\&A, 342, L32

Eberhardt, P., Meier, R., Kranowsky, D., \& Hodges, R. R. 1994, A\&A, 288, 315

Menten, K. M., Reid, M. J., Pratap, P., Moran, J. M., \& Wilson, T. L. 1992, ApJ, 401, L39

Minier, V., Booth, R. S., \& Conway, J. E. 2000, A\&A, 362, 1093

Norris, R. P., et al. 1998, ApJ, 508, 275

Schutte, W. A., et al. 1996, A\&A, 315, L333

Skinner, C. J., Tielens, A. G. G. M., Barlow, M. J., \& Justtanont K. 1992, ApJ, 399, L79

Slysh, V. I., Val'tts, I. E., Kalenskii, S. V., \& Larionov, G. M. 1999, Astronomy Reports, 43, 657

Slysh, V. I., Voronkov, M. A., Val'tts, I. E., \& Migenes, V. 2002a, Astronomy Reports, in press

Slysh, V. I., et al. 2002b, ApJ, 564, 317

Sobolev, A. M., Cragg, D. M., \& Godfrey, P. D. 1997, A\&A, 324, 211 